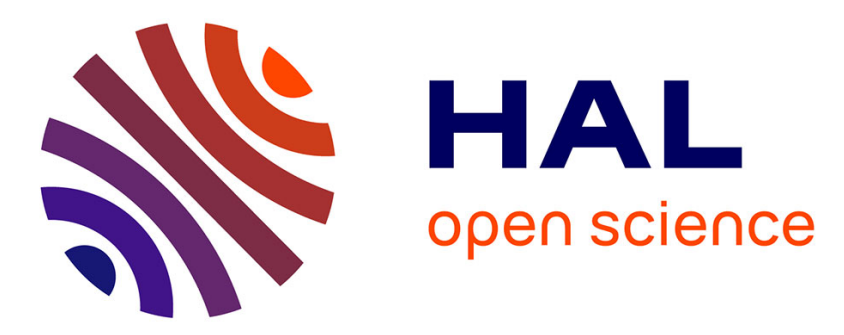

\title{
Im Dickicht der Sinne, ein melancholisches Gefühl. Betrachtungen zu Dezember von Alexander Kluge und Gerhard Richter
}

Hilda Inderwildi

\section{- To cite this version:}

Hilda Inderwildi. Im Dickicht der Sinne, ein melancholisches Gefühl. Betrachtungen zu Dezember von Alexander Kluge und Gerhard Richter. Herbert Holl, Clemens Pornschlegel, Vincent Pauval. Von Sinn(en) und Gefühlen, 5, V\&R unipress, pp.129-149, 2019, Alexander Kluge-Jahrbuch. hal02333379

HAL Id: hal-02333379

https://hal-univ-tlse2.archives-ouvertes.fr/hal-02333379

Submitted on 25 Oct 2019

HAL is a multi-disciplinary open access archive for the deposit and dissemination of scientific research documents, whether they are published or not. The documents may come from teaching and research institutions in France or abroad, or from public or private research centers.
L'archive ouverte pluridisciplinaire HAL, est destinée au dépôt et à la diffusion de documents scientifiques de niveau recherche, publiés ou non, émanant des établissements d'enseignement et de recherche français ou étrangers, des laboratoires publics ou privés. 


\section{Inhalt}

Einleitung: Sinn(e) und Zeit - Kluges Parteinahme für die Gefühle in

Anbetracht der Geschichte . . . . . . . . . . . . . . . 11

Alexander Kluge

Mit allen Sinnen sannen wir auf Rettung . . . . . . . . . . . . . . 17

Georges Didi-Huberman

Hunderttausend Milliarden Bilder . . . . . . . . . . . . . . . . . . . 19

Sven Hanuschek

Laudatio auf Alexander Kluge. Jean Paul-Preis 2017 . . . . . . . . . . 25

Alexander Kluge / Vincent Pauval

»Erzählen hat sehr viel mit Empathie zu tun» . . . . . . . . . . . . 33

Alexander Kluge

Ort der Einfühlung . . . . . . . . . . . . . . . 47

Ulrike Sprenger

Pikareskes Geschmackserlebnis und europäisches

Unterscheidungsvermögen: Ernst Opel an der Ostfront . . . . . . . . . 49

Alexander Kluge

Türkischer Honig . . . . . . . . . . . . . . . . 65

André Combes

Vom blinden Sehen bei Alexander Kluge . . . . . . . . . . . . . 67

DCTP - Ten to Eleven vom 2. Oktober 2017 (RTL) (Kluge / van Hemmen)

"Die Mathematik der sinnlichen Kraft« - Leo van Hemmen, Biophysiker 
Alexander Kluge

Scarpia als Männerkörper. Die tausend Seelen des Polizeichefs _ . . . . 95

Maguelone Loublier

Eine gespenstische Stimme geht um in Alexander Kluges Filmen . . . . 101

Vanessa Scharrer

Lichtfiguren. Die Plastizität des Lichts in Alexander Kluges

Minutenfilmen »Die sanfte Schminke des Lichts» _ . . . . . . . . . 115

Alexander Kluge

Großaufnahme eines Grasbüschels . . . . . . . . . . . . . . . . . 127

Hilda Inderwildi

Im Dickicht der Sinne, ein melancholisches Gefühl. Betrachtungen zu

Dezember von Alexander Kluge und Gerhard Richter . . . . . . . . . 129

DCTP - News \& Stories vom 3. August 2014 (SAT.1) (Kluge / Kiefer)

Ein Vormittag mit Anselm Kiefer - Besuch in seinem Atelier bei Paris . . 151

Helen Müller

Kooperation. Zur Schärfung eines Begriffs anhand von Alexander Kluges

jüngster Zusammenarbeit mit Georg Baselitz: Weltverändernder Zorn.

Nachricht von Gegenfüßlern . . . . . . . . . . . . . . . . . . . . . 169

Alexander Kluge / Vincent Pauval

Wenn Worte Bilder sehen . . . . . . . . . . . . . . . . . . . . 183

Vincent Pauval

Kommentar und Common sense als narrative Haltung. Zur Logik des

Elementaren bei Alexander Kluge . . . . . . . . . . . . . . . . . 203

Alexander Kluge

»Flüssigmachen« . . . . . . . . . . . . . . . . . . 221

Stefan Schweigler

Politiken der Wahrnehmung und queere Gefühle. Alexander Kluge und

Lilo Wanders . . . . . . . . . . . . . . . . . . . . . . . 223

DCTP - Ten to Eleven vom 22. September 1997 (RTL) (Kluge / Wanders)

»Ich sage niemals Mausi« - Lilo Wanders und das Hohelied Salomos . . . 243 
Martin Rass

Double blind - Alexander Kluges abarischer Punkt . . . . . . . . 253

Alexander Kluge

Das Gewicht des Gefühls, dort, wo es wenig galt . . . . . . . . . . . . 271

Melanie Konrad

Kinofizierungs- und Mediatisierungsverfahren. Zur Produktion von

Gefühlen und Geschichtlichkeit in Alexander Kluges Nachrichten aus der

ideologischen Antike . . . . . . . . . . . . . . . . . 273

Wolfgang Asholt

Die Herzlichkeit der Vernunft oder "was den Menschen im eigentlichen

Sinne menschlich macht . . . . . . . . . . . . . . . 289

Alexander Kluge

Ist Hilde unvernünftig ? . . . . . . . . . . . . . . . . . . . . . . . 303

Christian Wimplinger

Über Eigensinn, Arbeit und andere Zombiegeschichten. Enteignung des

Nah- und Fernsinns bei Negt und Kluge . . . . . . . . . . . . 305

Alexander Kluge

Mein wahres Motiv . . . . . . . . . . . . . . . 315

Rainer Stollmann

»Was der Mann für Unsinn macht» . . . . . . . . . . . . . . . . 319

Jean-Pierre Dubost

Desorientierte Orientierungen. Topographie und Navigation bei

Alexander Kluge . . . . . . . . . . . . . . . . . . 341

Alexander Kluge

Parade in der Sylvesternacht 1918 in Paris. Wie wir zu spät lernten, den subjektiven Eindruck auf dem Filmmaterial festzuhalten . . . . . . . 357

Alexander Kluge

Vorrede zu Chronique des sentiments - Livre II - Inquiétance du temps (P.O.L, 2018) . . . . . . . . . . . . . . . . . . . 361 
Alexander Kluge / Vincent Pauval

»Anwalt der dreizehnten Fee» - Über »Unheimlichkeit der Zeit» . . . . . 365

Alexander Kluge

Eingemachte Elefantenwünsche . . . . . . . . . . . 375

Birgit Haberpeutner

Der langrüsselige Atem der Rache . . . . . . . . . . . . . . . . . . 379

Clemens Pornschlegel

Verschönerungsarbeiten am Recht. Notiz zu Alexander Kluges Erzählung

»Bettine G.» . . . . . . . . . . . . . . . . . . . . . . . . . . . . 389

Herbert Holl

Zeitentzug, Sinnentzug $-»$ Sinnlich sein heißt leiden« . . . . . . . . . 401

Alexander Kluge

Rachegefühl als Freizeitthema . . . . . . . . . . . . . . . 421

Herbert Holl

Anita G.s Zeitfluxion . . . . . . . . . . . . . . . . . . . . . . 427

Kza Han

Pour Alexandra Kluge - Speculum animae /

Für Alexandra Kluge - Speculum animae . . . . . . . . . . . . . . . . 439

Rezensionen

Sylvie Le Moël

Kluges Pluriversum - zum Greifen nah . . . . . . . . . . . . . . . . 447

Winfried Siebers

Christoph Streckhardt, Kaleidoskop Kluge. Alexander Kluges Fortsetzung der Kritischen Theorie mit narrativen Mitteln, Tübingen: Narr Francke Attempto 2016. 451 Seiten (ISBN: 978-3772085888). . . . . . . . . . . . 451

Valentin Mertes

Dorothea Walzer, Arbeit am Exemplarischen. Poetische Verfahren der Kritik bei Alexander Kluge, Paderborn: Fink 2017. 214 Seiten (ISBN:

978-3-7705-6201-5). . . . . . . . . . . . . . . . . . . 459 
VeRZEICHNISSE 2017

Beata Wiggen

Verzeichnis der Kulturmagazine 2017

Winfried Siebers

Bibliographie zu Alexander Kluge 2017 . . . . . . . . . . . . . . 471

Autorinnen und Autoren . . . . . . . . . . . . . . . . 483

Buchanzeige: Philipp Ekardt, Toward Fewer Images. The Work of

Alexander Kluge . . . . . . . . . . . . . . . . . . . . . 493 
Hilda Inderwildi

\section{Im Dickicht der Sinne, ein melancholisches Gefühl. Betrachtungen zu Dezember von Alexander Kluge und Gerhard Richter}

In Dezember, dem gemeinsam mit Gerhard Richter $2010^{1}$ veröffentlichten Künstlerbuch, kontrastieren Kluges Geschichten mit den Landschaften Richters: "Das sind zwei Welten. Ich kann dem Affen Zucker geben. Ich kann dramatisieren. Richters Fotos hingegen, diese ruhigen, magischen Ewigkeitsbilder, lassen sich durch nichts erschüttern. ${ }^{2}$ Wie eine Art Flash News vollziehen die Texte Sprünge auf der Zeitskala - von 21.999 v. Chr. bis 31.12. 2009 - und bilden eine Sammlung von Lebens- und Geschichtsmomenten, zwischen bitterer Komödie und intimem Leid: Es scheint, als würden die Selbstbesinnung und die $\mathrm{Zu}$ rückgezogenheit der Berge in Sils Maria unentwegt vom Lärm der Welt erschüttert. Den Texten gegenüber befinden sich Bilder schneebedeckter Wälder, die von keiner, oder fast keiner, Anwesenheit gestört werden. Sie laden zur Kontemplation, wenn nicht sogar zur Andacht ein und betten den Betrachter in die langsame Zeitlichkeit unscheinbarer Veränderungen, eine Art Zeitlosigkeit. Die meisten dem Buch gewidmeten Rezensionen und Studien beginnen mit der Hervorhebung dessen, was auf den ersten Blick als zutiefst unzusammenhängend, sogar als Dissens erscheint, bekräftigt durch Kluges Worte. Diese Offensichtlichkeit ist jedoch zu kurz gesehen: Ein "gedehnter Blick « ${ }^{3}$ auf Richters Fotos enthüllt deren Unheimlichkeitspotenzial, ihre subtile Mischung aus Ausgeglichenheit (Ruhe, Magie, sagt Kluge), kalter Gleichgültigkeit und möglichem Schrecken, stets in Resonanz mit den Wendungen, den flüchtigen Launen und tiefgründigen Fragestellungen, die die Geschichten Kluges durchdringen.

Die folgende Analyse zielt auf die Übergänge von einer Empfindung zur

1 Alexander Kluge/ Gerhard Richter, Dezember, Berlin 2010. Im Nachfolgenden mit »D«abgekürzt.

2 "Gerhard Richter zeigt den Winter«, in: Rheinische Post, 21. 10.2010. [https://rp-online.de/kul tur/gerhard-richter-zeigt-den-winter_aid-12607875].

3 Diese Art Blick wird von Wilhelm Genazino 2004 in seinem gleichnamigen Werk, Der gedehnte Blick, aufgearbeitet. Es handelt sich um einen offenen und verlängerten, sperzeptiv-rezeptiven` Blick, der auf den Dingen verweilt, um ihr Aufblühen zu ermöglichen. Das fotografische Medium erscheint als quasi natürliche Ausweitung dieses Blickmodus. 
nächsten und die Umstände dessen, was ich »intermediale Präzipitation « nenne. ${ }^{4}$ Sie tut der evidenten Pertinenz anderer Lesearten, wie etwa einer vom Zufall ausgehenden ${ }^{5}$, keinen Abbruch, sie versteht sich jedoch als zugleich präziser und weitreichender. Der Meteorologie entlehnt, stellt der an die Empfindung gebundene Begriff der "Präzipitation" (Niederschlag) eine Möglichkeit dar, die zwei Ausdrucksweisen, Bild und Text, zu erfassen und zu zeigen, wie sich das Heterogene transformiert, wie es Sinn macht. Dieser Zugang nähert sich in mehreren Aspekten dem öko-kritischen Prisma von Urs Büttner ${ }^{6}$ an, aber sie orientiert sich im vorliegenden Fall mehr an Bildern des Waldes als an jenen der Kälte, um die Ketten des Sinns und die Vernetzung der Sinne nachzuvollziehen. Dem Lauf der Fotografien und Geschichten von Dezember folgend, liegt ihr die Hypothese zugrunde, dass sich nicht nur dieses, sondern die Werke von Kluge und Richter allgemein, um die Achsen Raum und Zeit kristallisieren, in einem Materialismus, der sich jeglicher Form von Hierarchisierung verweigert. Der Natur dieser Hypothese entsprechend soll meine Interpretation die Nähe zu einer solchen Hegemonie vermeiden, indem sie sich vor der quasi instinktiven Tendenz zur Homogenisierung des Heterogenen hütet.

4 Da mir die additive Perspektive einer Kopräsenz der Medien zu beschränkt erscheint, betrachte ich Intermedialität als einen Blickwechsel (Emmanuel Wallon) und in Bezug zum Raum: einen Raum, in dem es möglich ist, Elemente zu deponieren, um Prozesse der Formierung und Transformierung des Heterogenen im chemischen, ja meteorologischen Sinne, der Präzipitation, besser wahrzunehmen. Mehr noch als der Begriff der Träger (Éric Méchoulan) erlauben es die konkreten und dynamischen Begriffe des Raums, die konstitutiv für Kunst und Meinung sind, und der Präzipitation als Potentialisierung des Seins, die Bewegung der intermedialen Elaboration so nah wie möglich an den Schöpfungsprozessen, dem Leben der Künstler, im Laufe der Zeit und an den Orten der Schöpfung zu erfassen, indem die Praxen der Sinnstiftung, vor allem der Begriff der Grafie, ausgeweitet in seinen skripturalen, körperlichen und plastischen Bedeutungen, untersucht werden. Dies sind die Postulate der Habilitationsschrift, die ich zurzeit dem künstlerischen Dialog zwischen Kluge und Richter widme.

5 Maud Hagelstein/ Céline Letawe, »Alexander Kluge/Gerhard Richter. L'art contre le hasard«, in: Grégory Cormann/ Jeremy Hamers/ Céline Letawe, Lecteurs/Spectateurs d'Alexander Kluge, Cahiers d'Études Germaniques/69, Montpellier/Nizza 2015, S. 105-116.

6 Urs Büttner, "Nature Makes History: Narrating Nature in Gerhard Richter and Alexander Kluge's Dezember «, in: Fekadu/Straß-Senol/Döring, Meteorologies of Modernity. Weather and Climate Discourses in the Anthropocene, REAL - Yearbook of Research in English and American Literature: Vol. 33, Tübingen 2017, S. 217-233. Die ökokritischen Interpretationen finden ihren Höhepunkt in der Deutung von Gunther Martens, "Alexander Kluges literarisches Oeuvre als `Cli-Fi`«, in: Rainer Stollmann, Thomas Combrink, Gunther Martens (Hg.), Stichwort: Kooperation. Keiner ist alleine schlau genug, Alexander Kluge-Jahrbuch, Bd. 4, Göttingen 2017, S. $203 \mathrm{f}$. 


\section{Mystifizierung der Sinne, die Bilder im Bild}

Richters Bilder zeigen in Dezember unabänderlich Bäume und Schnee, im Schnee gefangene Bäume, aus größerer oder geringerer Nähe aufgenommen, hier und da mit einem Felschaos (22.12.) oder einer Kammlinie, die man mehr erahnt als sieht (25-26.12.). Es lässt sich nur schwer sagen, ob das Wetter gut oder schlecht ist. Horizonte sind selten und undeutlich. Sie tauchen nur auf sechs Fotos auf, vom 25. bis 30. Dezember, sowie auf dem Schlussbild. Der Boden ist vor dem 22. Dezember ebenso wenig präsent oder kaum identifizierbar. Die Zweidimensionalität, die Verdichtung des Raumes ohne Perspektive und die Vergrößerung des Astwerks vermitteln das Gefühl, dass man sich in einem ebenso figürlichen wie abstrakten Labyrinth befindet, in dem die Wirklichkeit auf Grund der Details und der Nachempfindbarkeit der Stoffe auf jeden Fall eine besondere Dichte gewinnt. In ihrer Entwicklung über Serien und Wiederholungen, scheinbar geringfügige Abwandlungen, die eine besondere Aufmerksamkeit und interpretative Arbeit erfordern, sind Richters Fotos mit Kluges Fragmenten vergleichbar. Sie wirken wie diese zugleich beruhigend und verwirrend. Ihr Auftauchen im Text gehorcht keiner systematischen Regelmäßigkeit, selbst wenn die Bilder die Erzählungen häufig einrahmen, ja verdecken. Am 8. und 30. Dezember sind zwei Fotos eingeschoben, darüber hinaus lädt die Anordnung der Bilder dazu ein, die Geschichten vom 20. und 21. als größere textuelle Einheit aufzufassen, welche die zentrale Figur des Geburtshelfers, die am symbolträchtigen 24. Dezember vorkommt, einführt; im Anhang »Kalender sind konservativ« erscheinen die Fotos paarweise oder zu dritt. Trotz dieser Disparitäten ist die Omnipräsenz der Waldbilder bemerkbar. Sie verleihen dem Text seinen Rhythmus, unterbrechen ihn und gewährleisten dennoch seine Kohäsion, sie statten ihn mit einem erstaunlichen, zugleich konkreten und abstrakten Fundament aus. »Richters Bilder unterlaufen die Zeit auf eine eigene Art und Weise, die nicht mit jener Kluges identisch ist; letzten Endes jedoch bekräftigen beide, Bild und Text, dieselbe Schlussfolgerung: Zeit ist Wetter. " Über die Polysemie des Wortes »temps « gibt die französische Sprache vorab die tiefen strukturellen Beziehungen zwischen »le temps qui passe« (die Zeit, die vergeht) und »le temps qu'il fait» (das Wetter, das herrscht) an. Die Dichotomie der Wörter »Zeit» (»time») und »Wetter» (»weather») steht jedoch nicht im Widerspruch zur Annahme, dass sie sich auf der paradigmatischen Achse überdecken. Von den 31 Geschichten, die der Monat Dezember (er)zählt, evozieren 21

7 LaRue, »Review of December by Alexander Kluge/ Gerhard Richter, Martin Chalmers (trans.) «, in: The Quaterly Conversation, No. 28, Summer 2012. "Richter's images have their own way of subverting time, which is not identical to Kluge's way; ultimately, however, both image and text reinforce the same conclusion: time is weather. " [http://quarterlyconversation.com/decemberby-alexander-kluge-and-gerhard-richter]. 
das Wetter, das in sechs davon das zentrale Thema darstellt. ${ }^{8}$ Die durch Echos und Schleifen gekennzeichnete narrative Struktur stellt eine deutliche Verbindung zwischen den Geschichten des 1., 4., 17., 23., 26. und 29. Dezember her.

Die Reflexion des Feldmarschalls Fedor von Bock in der einleitenden Geschichte zum 1. Dezember 1941 (»An sich brauchen wir [...] keine Waffen zur Bekämpfung der Russen, sondern eine Waffe zur Bekämpfung des Wetters. ${ }^{9}$ ) bildet die Grundlage für die Geschichte des 4., einen Wetterbericht: »die Ursachenkette, die im Dezember 1941 den Kälteeinbruch im Osten bewirkte ${ }^{10}$ und deren letztes Glied die Einführung der dynamischen Meteorologie darstellt. Tatsächlich scheint die Geotechnik mit den von Großbritannien während des 2. Weltkriegs eingeleiteten Operationen zur Impfung der Wolken über Deutschland gestartet worden zu sein. Kluge hingegen bevorzugt es, sie von der Nazi-Seite zu betrachten, wahrscheinlich aufgrund seines von Natur aus heterotopen Weltbilds, und um die Wettermanipulation nachdrücklicher mit dem zweifelhaften Traum der menschlichen Allmacht zu verbinden. Mit der Entwicklung von Mitteln zur Kontrolle von Temperatur, Niederschlag und Naturkatastrophen war der Mensch noch nie so nahe daran, sich zum Herrn der Welt aufzuschwingen. Auch wenn die dynamische Meteorologie für die Kämpfe an der Ostfront zu spät $\mathrm{kam}^{11}$, auch wenn sie den Lauf der Geschichte im Jahr 1941 nicht änderte, so schreibt der Glaube des Dr. Hofmeister in später umgesetzte Möglichkeiten selbst Geschichte, so dass man, wie oft in den Geschichten Kluges, auf eine Äquivalenz zwischen Tat und Erkenntnis - im doppelten Sinne von Wissen und Empfinden (Vorahnen) oder Gefühl - schließen kann. Diese Geschichte ist charakteristisch für das Klugesche Denken, sie »verkörpert eine Verbindung zu einer anderen möglichen Welt, an einem Punkt der Kausalkette, der noch nie zuvor als reale Interventionsmöglichkeit erkannt wurde. $\aleph^{12}$ Dasselbe Interpretationsschema findet Anwendung in Bezug auf das Scheitern der Klimakonferenz in Kopenhagen des 17. Dezember 2009, wobei die Analyse auf Größenverhältnisse ${ }^{13}$ ausgeweitet wird. Das Motiv »Die KLEINE EISZEIT«, das die Passivität der "Vertreter des UNO-Klimarates ${ }^{14}$ rechtfertigt, wird am 29. Dezember,

8 Nicht direkt mit dem Wetter verbunden sind die Geschichten des 8., 10.-11., 13., 16., 18., 21., 25., 27., 28. Dezember.

9 D, S. 7.

10 Ebd., S. 13.

11 Ebd., S. 14.

12 Büttner, "Nature Makes History«, S. 228. "This story is fascinating since it presents a junction into an alternate possible world at a point of the causal chain which had never before been noticed as a real option to intervene."

13 Die Größenverhältnisse, die Konfrontation des unendlich Kleinen und unendlich Großen, Lebensläufe und die Natur sind Leitmotive im Werk Kluges. Siehe dazu u. a. Alexander Kluge, Theorie der Erzählung - Frankfurter Poetikvorlesungen, 2 DVDs mit Beiheft, Berlin 2013. 14 D, S. 55. 
vermittelt über den nacherzählten Dialog zwischen Sylvie Charbit (Labor für Klima- und Umweltwissenschaften, Universität Versailles Saint-Quentin) und Alexej Tichonow (Abteilung für Paläontologie der Akademie für Wissenschaften, Sankt-Petersburg), in den großen Kontext der Eiszeit gestellt. Da es sich um reale Forscher internationalen Renommees handelt, ist anzunehmen, dass der Erzähler, Kluge oder Richter, tatsächlich einem Dialog dieser Art, einem ScienceFiction-Dialog, beigewohnt hat. Die Wettervorhersage fällt auf durch ihren retrospektiven Charakter - der nicht vor lückenhafter Kenntnis schützt -, auch durch ihre Langfristigkeit, d.h. viertausend Jahre großer Kälte nach Kalenderzeit. Der Mensch empfindet die Kälte unmittelbar, wenn die Temperatur unter Null fällt, ab »1 Uhr nachmittags« oder am "Spätnachmittag im Engadin im Dezember«, ein Ort, dessen Einzigartigkeit also auch daher rührt, dass er der gewöhnlichen Zeit der Zeitgenossen entzogen ist:

29. Dezember 21.999 v. Chr.: Eiszeit. Man muß sich diesen Höhepunkt der Kälte (noch immer leben wir in der gleichen Periode der Eiszeit, aber nicht in der Großen, sondern der Kleinen) klimatisch so vorstellen wie einen Spätnachmittag im Engadin im Dezember, sagte der wissenschaftliche Sekretär des Mammutkomitees der Russischen Akademie der Wissenschaften Alexej Tichonow. Nicht kälter? Fragte Sylvie Charbit zurück. Kalt genug, wenn Sie kein Heizmaterial in der Nacktsteppe finden und Wohnsitze fehlen.

Eine Wettervorhersage hätte in jenen Tagen so gelautet, fuhr der Russe fort: Europa liegt seit zwei Jahren unter einer Hochdruckzone. Der stetige Wind, der vom Packeis herweht, bringt sehr kalte, trockene Luft in die Region. Ein Ende der extremen Trockenheit, des stetigen Winds von Nordost, der viel Staub mit sich trägt, ist in den nächsten 4000 Jahren nicht zu erwarten. Ab 1 Uhr nachmittags sinkt die Temperatur unter den Gefrierpunkt. Und über die Trockensteppe, die es heute nicht mehr gibt (mit ihren Gräsern, nahrhaften Kräutern, aber keinen Bäumen), huschten die Menschen? Unsere Vorfahren, antwortete Tichonow, »huschten « nicht, sie suchten, fahndeten und jagten im Wettlauf mit dem Tod. Wenn sie nicht schnell etwas fanden, verhungerten sie. ${ }^{15}$

Man ist verführt, diese Bilder als Embleme zu betrachten. Einerseits zeigen sie die durch die Landschaften Richters illustrierte Eiszeit und die Vormacht der Natur, ihre düstere Schönheit und eine Art Zeitlosigkeit; andererseits die Sterblichen auf der Lauer in den `Ausgrabungs-Texten $८$ Kluges, die Sinnpartikel und die Suche des Sinns. Letztere (Menschen wie Texte) eint die Weigerung zu "huschen «, zu schnell zu vergehen, lautlos, unbemerkt.

Der Text des 29. Dezember stellt die scheinbare Ruhe der Natur nicht den schrillen Dissonanzen menschlicher Kämpfe gegenüber. Er konfrontiert auch nicht die unterschiedlichen Zeitschichten, er hütet sich vor definitiven oder eindeutigen Interpretationen. In diesem Zusammenhang erscheint Dezember wie eine feine, präzise Abwandlung der umfangreicheren Studien, die Richter

15 D, S. 90. 
und Kluge den Möglichkeiten widmen, weitläufige Gesamtheiten wie Wald und Kälte darzustellen oder spürbar zu machen, jeder mit dem ihm eigenen Ausdrucksmittel, zwischen Ereignis und Dokument ${ }^{16}$ - an der Grenze des Figürlichen und Abstrakten bei Richter, zwischen Poesie und Theorie bei Kluge.

Es scheint übertrieben, wie Marius Hulpe zu behaupten, dass die einzige Verbindungslinie zwischen den Fotos und den Texten der Monat Dezember sei, dass der `Dezember das einzige Thema sei, das die Künstler diszipliniert verfolgten, "so diszipliniert, dass es teilweise etwas von einer monumentalen Installation in Buchform hat, was sie hier nebeneinanderlegen. ${ }^{17}$ Angemessener erscheint die Idee, die verschneiten Landschaften Richters als "winterliche Versuchsanordnungen ${ }^{18}{ }^{\mathrm{zu}}$ betrachten, deren Variationen, so geringfügig sie auch sein mögen, zugleich Strukturmuster freilegen, wie Sinnestäuschungen wirken, und somit an Mystifikationen grenzen. Diese gilt es zu analysieren, angefangen bei Richters Umgang mit den emblematischen Motiven des konkreten Raumes und der Bildsymbolik um Weihnachten. Ob für sich selbst oder in der reziproken Erhellung mit den Texten, zeigen die Fotos in Dezember das Bild allgemein als Ort, an dem sich die Anwesenheit anderer Bilder manifestiert. Das Bild ist dort Absorption und Transformation eines anderen Bildes, eingebunden in ein inter-ikonisches Netzwerk und in Spiegelungen, in denen das Explizite vom Impliziten abgelöst wird. Aus diesem Grund bietet sich eine intermediale Interpretation an. Sie erlaubt jene sinnstiftenden Effekte herauszufiltern, die durch die Aufnahme von Fotos in ein narratives Konglomerat produziert wer-

16 Kluges und Richters Verhältnis zum Dokument ermöglicht es, die Bedeutung, die die Kombination Vorstellungskraft/kritische Vorgehensweise für sie hat, nachzuvollziehen. Ihr Zugang ist mit jenem von Robert Escarpit vergleichbar, der das Dokument als glückliches "Anti-Ereignis« definiert, das man vor allem in seiner Materialität erfassen müsse, wie eine Gegebenheit oder feste Spur. So kann man es lesen und ihm einen Sinn geben, trotz der Dekontextualisierung der Information. Es erscheint unumgehbar für den, der ein Ereignis rekonstruieren oder erzählen will. Bei Kluge und Richter ist das Dokument meist der kürzlichen Geschichte oder Nachrichten entlehnt, die es als Meinungsvektor benutzen. Diese Art Dokument setzt dem Risiko einer zu großen Nähe und der Kurzsichtigkeit aus, dennoch spielt es bei den Künstlern eine strukturierende Rolle, auf der Ebene der Schöpfung ebenso wie des Gedächtnisses (und damit, der Überlieferung). Über das Schreiben und die Malerei streben die zwei Künstler mangels Sinns die Stiftung von Wissen an: "Die Information ist eine negative Menge, die dem Geist durch das Ereignis zugetragen wird, wenn es von der Zukunft in die Vergangenheit übergeht. Das Wissen ist eine recht stabile Konstruktion, um den Ablauf der Zeit zu bremsen und im Maßstab eines Menschenlebens verfügbar zu bleiben, beweglich genug, um zu fortlaufend neue Antworten aufzunehmen und sie in lesbare Strukturen einzufügen«. Robert Escarpit, Théorie générale de l'information et de la communication, Paris, Hachette, 1976, S. 59f.

17 Marius Hulpe, »Alexander Kluge/Gerhard Richter: Dezember. Historisch, ahistorisch?«, in: Litmag, 27.4.2011. [http://culturmag.de/rubriken/buecher/alexander-kluge-gerhard-rich ter-dezember/24770].

18 Ebd. 
den, während das Ensemble sich zunächst durch seinen disparaten und geheimnisvollen Charakter auszeichnet.

\section{Sinn, Sinne und Gefühle in Richters Wäldern}

Mit Dezember setzt der Maler auf kooperative Art und Weise die Arbeit, die 2008 zur Erscheinung seines Künstlerbuches Wald ${ }^{19}$ führte, fort. Richter spricht dabei von einem »Alterswerk«:

Ja doch, denn die Faszination, die ich damals empfand, also dass ich wie vernarrt dieses Gestrüpp, diese Anblicke abseits der Wege suchte und als so schön und tröstlich empfand, dass ich sie immer wieder fotografierte, das hat schon was mit Alter und Altersängsten zu tun. Was auch immer, es war ein sehr schönes Projekt für mich. Es kann sein, dass es mein liebstes Buch ist. ${ }^{20}$

Die Bilder von Laub- und Nadelbäumen, von dichten oder lichten Wäldern, mit und ohne Schnee, systematisieren sich im Werk Richters seit Ende der 1960er Jahre. Er fotografiert sie allerorts, nicht nur in Hahnwald, dem Viertel Kölns, in dem er seit 1992 wohnt, sondern auch an seinen bevorzugten Urlaubsorten, vor allem auf Korsika und in der Schweiz, in Davos und besonders in Sils Maria. Die Serien fotografischer Studien finden ihre Berufung als Richter eine Nummer des Fachmagazins Waldung (1/2006) entdeckt, das den Anspruch einer »interdisziplinären« Vision des Waldes erhebt: "Der Wald ist nur interdisziplinär zu begreifen: Als Erinnerung an Märchen und Wanderungen. Als Teil unserer Heimat und Landschaft. Als Projektionsfläche von Wünschen und Ideologien. Als komplexer Lebensraum und Rohstofflieferant. « ${ }^{21}$ Wald (2008) verlängert dieses Projekt und durchkreuzt es zugleich über die Zersetzung der an die Welt des Waldes gebundenen Diskurse und Topoi, indem diese mittels Zufallsgenerator aleatorisch angeordnet werden. Die Atmosphäre des Textes verändert sich jedoch nicht wesentlich, da das semantische Feld des Waldes aufrechterhalten wird, sodass der Maler feststellt: "Manchmal kann ich das mit einem gewissen Wohlgefallen selbst lesen. « $^{22}$

Der Text entspricht einer minutiösen und höchst komplexen Konfiguration, während die 285 Fotos, die das Buch umfasst, auf einfache Weise, einer objektiven und neutralen Typologie folgend - nach Dicke des Astwerks, der Anord-

19 Richter, Wald, Köln 2008.

20 Obrist/Schwarz, Gerhard Richter. Bücher, hrsg. von Dietmar Elger, Staatliche Kunstsammlungen (= Schriften des Gerhard Richter Archiv Dresden, Bd. 11), Dresden 2013, S. 89.

21 Das Magazin wird vom Künstler und Förster Nikolaus Theile infolge eines Forschungsprojektes gegründet. [https://www.khm.de/studentische_arbeiten/id.12806.waldung/].

22 Obrist/Schwarz, Gerhard Richter. Bücher, S. 91. 
nung horizontal liegender Stämme und der Neigung diagonal oder vertikal wachsender Bäume - arrangiert sind. Man denkt an die deutschen "Baumbücher " Ende des 19. und Anfang des 20. Jahrhunderts, mit dem Unterschied, dass es hier nicht um eine Inventarisation der Exemplare geht. Richters Bäume zeigen sich in einer Art vertrauter Banalität. Dennoch erzeugt die Serie einen Eindruck der Dichte, die zahlreichen und wiederkehrenden Bilder bilden einen geeigneten Raum, um sich von üblichen Anhaltspunkten zu lösen und zu verlieren. Aber in diesem Wald verliert man sich nicht auf dieselbe Weise wie in jenem Wald von 1966, in dem das Liebespaar, das sich dort versteckte und in jeder Hinsicht vom rechten Weg abkam, einen fragwürdigen Unterschlupf bot. Nun gibt sich der Maler einer Art Kontemplation hin, einer besonderen Methodik der Landschaftsstudie, die zur Abstraktion führt: In den ersten gemalten Studien, wie Baumgruppe $(1970,1987)$ und Bühler Höhe (1991), ergibt sich der abstrakte Charakter der Landschaft aus ihrer Verfremdung. In späteren Fotoserien zeigt Richter auf direkte Weise konkrete Abstrakta und die paradoxen Eigenschaften der Landschaft. Seit den 1970er Jahren behauptet der Maler gerne, dass er, was ihre "Organisation « betrifft, keinen Unterschied zwischen abstrakten und figurativen Bildern mache:

Die Mittel, die man zur Organisation verwendet, sind dieselben: dieselbe Struktur, dieselben Kontraste, solche Dinge. Aber es gibt einen Unterschied in dem, was ich Klima nenne. Die Landschaften, zum Beispiel, sind ruhig, sentimental. Die abstrakten sind emotionaler, aggressiver. Diese Unterschiede suche ich im Klima. ${ }^{23}$

Diese Aussage wird unter anderem von der abstrakten Serie Wald (2005) bestätigt. Die zwölf Großformate sind von denselben Waldfotos wie das Künstlerbuch des Jahres 2008 inspiriert, aber "obwohl sie den gleichen Titel haben, hat Wald bei den Gemälden nichts mit einem konkreten Wald zu tun. ${ }^{24}$ Wenn auch legitim, so ist es doch nicht zwingend notwendig, wie Mark Godfrey eine Verbindung zwischen dieser Serie und den Birken von Birkenau ${ }^{25}$ herzustellen, um die dunklen Hintergründe hinter den Lichtblitzen und der Flüssigkeit der Materie, die fallenden Regen evoziert, zu erkennen. Die Baumvorhänge des Künstlerbuchs produzieren unweigerlich einen weniger spektralen Eindruck als die abstrakten Bilder und man kann sie zweifellos als »ruhig» bezeichnen. Aber auch in diesem Werk ist die Form fragmentarisch, diffus. Sie erhebt keinen Anspruch auf Regelmäßigkeit oder Klarheit, sie strebt nicht danach, Inkompa-

23 »Interview mit Anna Tilroe 1987«, in: Text 1961 bis 2007. Schriften, Interviews, Briefe, hrsg. von Dietmar Elger und Hans Ulrich Obrist, Köln 2008, S. 201. Vgl. »Interview mit Irmeline Lebeer 1973«, in: Ders. S. 72-79, 83.

24 Obrist/Schwarz, Gerhard Richter. Bücher, S. 91.

25 Mark Godfrey, A Curtain of Trees, Tate Modern, Londres 2011. [https://www.gerhard-richter. com/fr/videos/talks/a-curtain-of-trees-50]. 
tibilitäten künstlich aufzulösen. Darin besteht die Nähe zwischen den konkreten Motiven auf den Fotos und den abstrakten Bildern. Diese manifestiert sich auch in der Wahrnehmung des Betrachters, denn Abstrakta wie Konkreta beeindrucken durch ihre hypnotische Wirkung, durch die Spannung, die sie dem Blick aufzwingen, zwischen extremer Konzentration und Schwanken oder Aufhebung. Gemeinsam sind ihnen des Weiteren der methodische Zugang und die Suche nach Exaktheit, die der Maler schon 1987 erwähnt:

Manchmal wirken Ihre abstrakten Bilder wie Landschaften. Suchen Sie in der Abstraktion erneut den Realismus?

Ich glaube, ich suche Genauigkeit. Mein Werk hat insoweit mit der Realität zu tun, dass ich möchte, dass es eine ähnliche Genauigkeit hat. Das schließt nachmalen aus. In der Natur stimmt immer alles: Die Struktur ist richtig, die Proportionen stimmen, die Farben passen zu den Formen. Wenn man das nachmalt, wird es falsch. ${ }^{26}$

Diese Suche nach Stimmigkeit und Genauigkeit ist umso anspruchsvoller, als es Richter darum geht, Ungewissheit wiederzugeben. Was sich indirekt aus den Fotografien ablesen lässt, verbunden mit den "Altersängsten«, erscheint auf den abstrakten Bildern als essentielle und vertraute »Ratlosigkeit«, ja Ohnmacht, »so als hätte man sich verirrt. Geschildert wird ja eher das Gefühl, das man in dem Wald hat, den man nicht versteht. ${ }^{27}$ Die Vertrautheit entsteht aus dem konkreten Wald, in dem man stundenlang im Kreis laufen kann, ohne Orientierung zu finden. Was sich darin ausdrückt, ist »ein melancholisches Lebensgefühl $\aleph^{28}$, das Richter mit dem romantischen Geist verbindet, a fortiori in seinen abstrakten Bildern. Die Fotos des in Hahnwald als Park angelegten Blätterwaldes (keines vermeintlich deutschen Nadelwaldes) verweisen mehr auf eine soziale Konstruktion des Natürlichen als auf die romantische Suche nach einer Nationalkultur. Vermutlich in diesem Sinne stellt Cord Riechelmann in Richters Künstlerbuch eine unerwartete fast antideutsche Haltung fest. ${ }^{29}$ Vielleicht wird dieses Urteil der Bedeutung der Buchen- und Eichenhaine von Goethe, und der historischen Rolle, die der Linde und der Eiche für Jakob Grimm innewohnt, nicht ganz gerecht, aber in der Tat situiert sich Richter in einem anderen post-romantischen Raum. Sein Wald ist nicht großflächig, man sieht davon nur Segmente, einfache Stämme ohne Wurzeln oder Wipfel: »Er verweigert den Aufblick auf den Wald. Das Kronendach des Waldes ist dem Spaziergänger Richter nicht

26 "Interview mit Anna Tilroe 1987«, S. 200.

27 Obrist/Schwarz, Gerhard Richter. Bücher, S. 92.

28 Ebd.

29 Cord Riechelmann, »Wider den deutschen Forst «, Buchkritik, in: taz, 23. 10. 2009, S. 16. »Das ist, wenn es um den Wald geht, weniger deutsch, als man bei Richter erwartet hätte. [...] Richter trifft mit der Wahl seiner Bäume aber noch ein paar andere, fast schon antideutsch zu nennende Entscheidungen.» 
zugänglich. Er bleibt am Boden. $\aleph^{30}$ Über die schrumpfende physische Welt des Alters hinaus, verkörpert das Buch Wald (2008) das Bemühen um Verankerung in einer neutralen Materie. Die Begrenzungen des Rahmens und des Blickes lassen sich positiv und nicht nur als Baum, der den Wald verdeckt, deuten.

Die Fotos in Dezember weisen Analogien zu jenen des Buches von 2008 auf, man kann sie als winterliches Pendant zu dieser Studie herbstlich-winterlicher Wälder und ihrer Lichtspiele betrachten. Die Detailaufnahmen sind aus noch geringerer Nähe aufgenommen und in zahlreichen Fällen sind es weniger die Stämme als das Astwerk, dem Richters Interesse gilt. Es vollzieht sich ein Wechsel der Größenverhältnisse, der auf den Pfad des Kleinen und unendlich Kleinen führt, wobei dennoch wahrnehmbar ist, dass der Wald in dieser Serie riesig und mysteriös ist. Die Ansichten von eingeschneiten Tannen evozieren Höhenlagen und intensivieren den Eindruck einer abgeschiedenen, dichten, ja undurchsichtigen Welt, die verunsichert, bezaubert oder beunruhigt, in Frage stellt. Dezember beginnt und endet mit Fotos von Richter und suggeriert damit, dass für den Künstler und seinen Koautor Bilder stets das letzte Wort haben: "Das sind eigentlich Erzählungen. Worte, in ein Bild gekleidet. ${ }^{31}$ Das Schlussfoto ist bemerkenswert, es spielt ganz offensichtlich mit den grafischen Topoi der allegorischen Darstellungen der vergehenden Zeit und des Lebens als zu durchlaufendem Weg. Die Bildausschnitte fordern dazu auf, dieses Foto nicht vorschnell als klassische Figuration zu lesen.

Im rechten oberen Viertel befindet sich eine überhängende Straße, gesäumt von hohen, potenziell gefährlichen Bäumen, bedeckt mit einer dünnen Schneeschicht mit mehr oder weniger tiefen Reifenspuren, abgegrenzt von einer Leitplanke und durch eine Haarnadelkurve abgeschnitten. Links zeichnet unberührter tiefer Schnee eine wattige Talmulde mit einem Pfad, der sich durch die feinen Baumgruppen schlängelt, bevor er in eine unbestimmte Ferne abtaucht. Hinter dem bräunlichen Schimmer der Baumspitzen am Horizont nimmt dieser unbestimmte schwindelerregende Raum fast die gesamte obere Hälfte des Fotos ein. Auffallend sind das Gleichgewicht der Komposition und die Graunuancen; die Disparität der vertikalen Flächen erscheint auf gewisse Weise durch den Parallelismus der offenen Wege im Schnee kompensiert. Hier und da eine Hütte oder ein Haus, die man nach und nach ausmacht - das sind die Spuren jener diskreten menschlichen Anwesenheit, die die Reifenspuren erahnen lassen. Das Foto erlaubt nicht nur eine symbolische Leseart, sondern setzt diese geradezu voraus. Man kann darin zugleich die Suggestion paralleler Lebensläufe mit ihren jeweiligen Mäandern und Wechselfällen sehen sowie die Darstellung von

30 Ebd.

31 «Erzählungen haben den Vorteil, dass sie blind sein dürfen." Ein Gespräch mit Alexander Kluge, in: VOLLTEXT 9, Okt/Nov 2003. 
Langzeit und Kurzzeit und das Hin und Her zwischen ihnen, jene eines mehr menschlichen und eines anderen mehr natürlichen Weges, des Wendepunkts des Alterns, eine Art und Weise, einen Schlusspunkt zu setzen, wobei gleichzeitig andere Räume, in denen sich andere Zeiten und neue Geschichten entfalten können, eröffnet werden. Aufgrund seiner narrativen Struktur und seiner Suggestionen kann man dieses letzte Foto als Emblem des gesamten Buches in seiner Kombination aus Text und Bild interpretieren. Der Herausgeber der englischen Fassung hat es übrigens als Titelbild der Neuauflage des Werks $2017^{32}$ gewählt, weil es zunächst weniger eintönig, unmittelbarer bedeutsam erscheinen kann, weil es auch die Tonalität der zweideutigen und melancholischen Träumerei des Buches von Kluge und Richter angibt.

Das erste Bild des Buches ist von ganz anderer Gestalt: wenig Boden, noch weniger Himmel, Vorhänge aus verzweigtem Astwerk, von einem Schneemantel bedeckt. Im Vordergrund heben sich zwei zarte Sträucher auf dornigem Hintergrund ab. Auf ihren Blättern befinden sich ein paar Flecken in intensivem Braunrot, wie ein Überbleibsel der vorangehenden Jahreszeit. Sie kündigen die Zwillingsbäume des eingeschobenen Fotos in der Geschichte des 30. Dezember an, von dem man auch ein Exemplar am 25. Dezember findet. Man kann nicht umhin, darin eine Anspielung auf das zu sehen, was Kluge und Richter vereint. Es handelt sich um ein Urbild, denn die hauchzarte und zugleich dichte Architektur, die dieses Eröffnungsbild verkörpert, wird in mehr oder weniger bemerkbaren Variationen, am 1.-3., 6.-9., 10.-11., 15.-19., 31. Dezember reproduziert. Richter fotografiert primär Lärchen, diesen einzigartigen Nadelbaum, der seine Nadeln im Oktober verliert. Andere Spezies von Nadelbäumen und dickerer Schneeschicht erscheinen insbesondere in "Kalender sind konservativ«. Die Behauptung, dass Richter jeden Tag ein neues Foto aufgenommen hätte, ist wohl seinem scherzhaften oder mystifizierenden Geist geschuldet: Gewisse Fotos wurden offensichtlich unmittelbar nacheinander aufgenommen (zum Beispiel die Totale aus Vogelperspektive vom 26. bis 29. Dezember); von den Aufnahmen verschlungenen Astwerks kommt mindestens eine in unterschiedlicher oder vergrößerter Ausrichtung noch einmal vor, am 10., 16. und 18.-19. Dezember. Auf diese Weise gibt sich der Fotograf im Off zu erkennen, gleich einem Forscher, der Räume und isomorphe Gesamtheiten konstruiert, deren manchmal winzige Relief-Effekte Auskunft über die Beschaffenheit der Wirklichkeit geben und den Blick stimulieren. Dieser wird zur Fokussierung angeregt, zum Ortswechsel durch das Foto und dem Hinübergleiten von einem Foto zum nächsten, um die versteckten Details und die Leserichtung zu erfassen. Ob es sich um Landschaften in Totale oder fraktale Architekturen handelt, der Betrachter wird von der wiederkehrenden und hypnotischen Natur der Auf-

32 December, translated by Martin Chalmers, University of Chicago Press 2017 [2012]. 


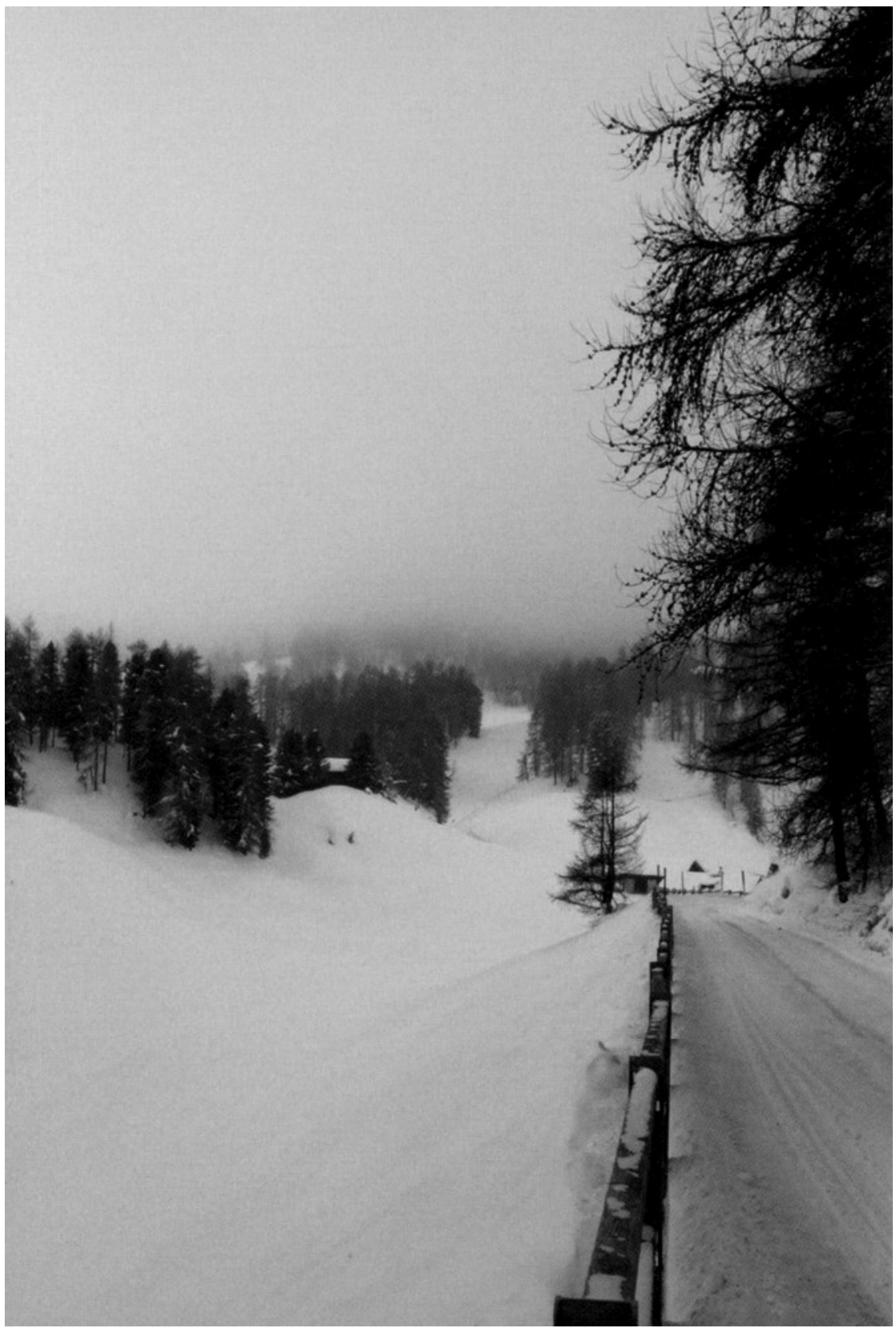

Abb. 1, Dezember, S. 126. @ Gerhard Richter 2018 (04072018) 
nahmen, die die Zeit auf kontemplative Weise takten, in den Bann gezogen. Er wird von der besonderen Qualität dieser "ruhigen, magischen Ewigkeitsbilder « angesprochen, die nichtsdestoweniger "ein Dickicht" multipler und düsterer Suggestionen darstellen:

Was sehen Sie in Richters Bildern?

Ich finde die Bilder mit den farbigen Blättern berührend. Ich deute die Verschränkung der Bäume im Engadin als Dickicht. Das erinnert an die Hecken von Dornröschen. Da spielt die dreizehnte Fee eine Rolle. Sie wurde ausgeschlossen. Daraufhin hat sie das ganze Schloss in Schlaf versetzt. Auch das ist im Dickicht der Bilder enthalten. Aber ich würde mich hüten, Richters Bilder mit Worten zu deuten. ${ }^{33}$

\section{Dickicht der Gefühle, Sinn für Musik und Sinnkonstellationen bei Kluge}

In dieser zugleich präzisen und vagen Antwort greift Kluge auf vorsätzlich lose miteinander verbundene Emotionen und Symbole zurück, nicht nur, um die fundamentale Ambivalenz und die allgemeine Tonalität der Bilder von Dezember spürbar werden zu lassen, sondern auch in Bezug auf das Wesen Richters: das eines "Bildermachers ${ }^{34}$. Der Autor nimmt dennoch wieder auf, was man als eine Art Lektüreschlüssel verstehen kann, der im Prolog jener Geschichte offenbart wird, die das Werk am 31. Dezember 2009 provisorisch schließt. Unter dem Titel "Die Macht der >Zeit«" führt das Ende des Textes die Figur von Mönch Andrej Bitow und die zugehörige Reflexion über Kalenderdaten ein. Der Anfang »EINDRUCK VON UNDURCHDRINGLICHKEIT « ist ein kurzer, indirekter, über die Überlagerung von Bildern vollzogener Kommentar des gegenüberliegenden Fotos. Bei Kluge lässt das Flechtwerk der Äste Kindheitsbilder aufkommen, die einerseits mit der Welt des Märchens, andererseits mit der des Krieges verbunden sind. Das Motiv des undurchdringlichen (auch unentwirrbaren) Waldes, der auch das Schloss Dornröschens umringt, wie man es zum Beispiel auf den Illustrationen von Otto Ubbelohde, Julius Diez oder Wilhelm Jordan sehen kann, deckt sich mit dem der militärischen Verhaue, und zwar durch den Neologismus (es sei denn, es handelt sich um einen Tippfehler) »der Verhak«, der zugleich ein »Verhaken« und einen aus Ästen und Bäumen errichteten Schutzwall zu sehen und zu hören gibt. ${ }^{35}$

33 "Gerhard Richter zeigt den Winter« (Anm. 2).

34 Kluge, Gerhard Richter: Bildermacher. dctp-tv, Ten to Eleven vom 19. März 2013: "Ich sollte mich nicht mehr Maler, sondern Bildermacher nennen."

35 Die "réseaux de frise» der französischen Übersetzung wollen das Bild der »chevaux de frise" (»Friesische, bzw. Spanische Reiter «) heraufbeschwören, die unter anderem während des 2. Weltkriegs die Strände spickten, wobei sie durch die Einführung des Wortes "réseau« 


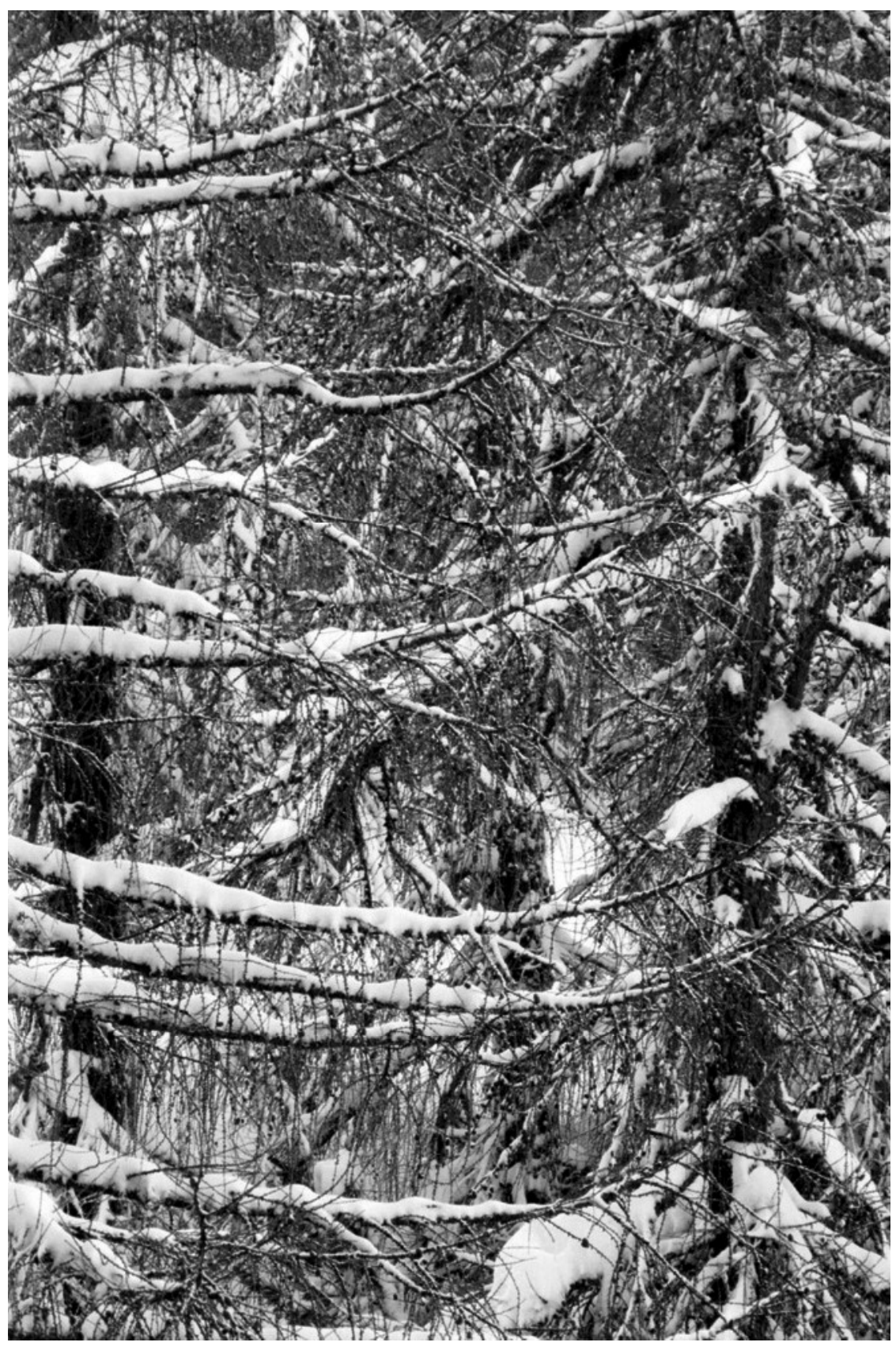

Abb. 2, Dezember, S. 99. () Gerhard Richter 2018 (04072018) 
31. Dezember 2009: EINDRUCK VON UNDURCHDRINGLICHKEIT. In der Sammlung der Brüder Grimm wird erzählt, daß es nur zwölf Gedecke gab für die zwölf »weisen Frauen des Landes«. Die 13. Fee wurde nicht eingeladen. Ähnlich wie bei der Havarie von Tschernobyl oder dem Sturz des Bankhauses Lehman Brothers die Fakten ausgegrenzt werden, die wenig später Unglück bringen. Die 13. Fee aber rächte sich, indem sie das Schloß und das Reich auf eintausend Jahre in Schlaf versetzte.

Zugleich umgab sie das Schloß mit einer Hecke aus Bäumen und Gestrüpp, das Gezweig ineinander verschachtelt, einen »Verhak « bildend. Schnee lag auf dem Geäst. Es entstand der Eindruck von Undurchdringlichkeit. In der Lebenspraxis aber zeigt sich, daß am Boden eines solchen Geästs ein Weg durch leichten Schnee zu finden ist. Man muß nur das Bild bis nach unten verfolgen, wo auf einem Quadratmeter Erde Milliarden Milben siedeln. $^{36}$

Der Bezug auf die Brüder Grimm evoziert unmittelbar eine kulturhistorische, intersubjektive und transpersonelle Sphäre, die an die deutsche Seele gebunden ist, der die Wälder zugleich Brut- und Zufluchtsstätte sind. Dem Gesetz der Märchen folgend, schafft Kluge auch einen gemeinsamen psychischen Raum für menschliche Nachlässigkeiten, Illusionen und Ängste, ebenso äußere wie innere Dramen. Die dreizehnte Fee verkörpert dort eine uralte Form der Überlieferung und einer zwiespältigen Tradition, die schmerzhafte Veränderungen oder unheilvolle Zwischenfälle erzeugt. Der Klugesche Synkretismus vereint in dieser Rächerfigur die Figur der letzten weisen Frau und der bösen Fee des Märchens, die so an Kraft und Ambiguität gewinnen. Wie gewisse radioaktive Teilchen löschen ihre vereinten Kräfte das Leben aus, versetzen es in einen nicht hundert-, sondern tausendjährigen Schlaf. Auf diese Weise bieten sie dem Leben aber auch einen Rückzugsort. Die Aktualisierung des Märchens und seiner Symbole, die das Unbewusste ansprechen, liefert so das Analysesubstrat einer komplexen Realität. Sie ermöglichen auch das Eindringen in die Bilder Richters, ausgehend vom Motiv der Hecke aus Bäumen und Sträuchern mit ihren verschlungenen Ästen. Der Eindruck der Undurchdringlichkeit wird durch den Beobachter aufgelöst, dem es gelingt, sich einen Weg durch den Wald zu bahnen, indem er "das Bild bis nach unten verfolgt«, um sich unter die Milben zu mischen, deren Larven das Holz aushöhlen.

Über die Homonymie mit »scierons« schlägt die französische Übersetzung von »Milben« eine ideengeschichtliche Schneise. Für den kultivierten französischen oder deutschen Leser öffnet der "ciron" die Tür zur Welt Montaignes. Er ruft die berühmten Sprüche der »Apologie für Raimond Sebond (Essais, II. 12) in Erinnerung: »Pareils appétits agitent un ciron et un éléphant.» (»Ein Wurm,

(Netz) eine positive Deutung suggerieren, um den essentiell dualen Charakter der Fotos von Richter wiederzugeben, zu denen man mit der Erwähnung des im Märchen nicht explizit erwähnten Schnees unbewusst hinübergleitet. Kluge/Richter, Décembre, übersetzt von Hilda Inderwildi und Vincent Pauval, Zurich-Biel/Bienne-Berlin 2012. 36 D, S. 98. 
und ein Elephant haben einerley Begierden.«), »L'homme est bien insensé. Il ne sçauroit forger un ciron, et forge des Dieux à douzaines." (»Der Mensch ist sehr unbesonnen. Er kann nicht eine Milbe machen, und macht ganze Dutzend Götter.«). Mit der Anspielung auf Montaigne sucht Kluge hier nicht vorrangig, den menschlichen Dünkel anzuprangern oder die Fundamente der Religion zu untergraben, selbst wenn er im zweiten Teil seiner Geschichte die Zäsur, die die Religion mit der Einführung der Zeit nach $\$ 6000$ Jahre[n] Vorgeschichte« geschaffen hat, ironisiert. Er nimmt die Milben und den Elefanten Montaignes in sein Bestiarium auf als konkrete Symbole zweier Extreme auf der Skala der Kreaturen, die sich in Ansprüchen wie Gefühlen generell kreuzen. 1968 inszeniert Kluge in seinem zweiten Spielfilm, Die Artisten in der Zirkuskuppel: ratlos der bei der Mostra Venedig den Goldenen Löwen bekommt und Kluge zur Leitfigur einer Strömung macht, zu der auch Wim Wenders, Volker Schlöndorff, Werner Herzog oder Rainer Werner Fassbinder gehören -, den Elefanten als Instrument einer Zirkusreform, deren Ziel es ist, das Publikum an dessen eigene Empfindungen heranzuführen, indem sie es dem Blick eines Dickhäuters aussetzt. In ihrer bemerkenswerten Analyse betrachten Grégory Corman und Jeremy Hamers die Figur des Elefanten bei Kluge als mögliches Bindeglied zwischen Film, Gefühl und politischem Engagement. ${ }^{37}$ Im Rahmen einer Theorie des Gefühlserbes situieren sie den Elefanten auf der Linie, die vom enttäuschten Urvertrauen zu einem meist passiven aber radikalen Widerstand führt, der auf die Praxis der kritischen Theorie des späten Adorno verweist. Zunächst als Werkzeug des Scheiterns dargestellt, da er auf keinen Befehl reagiert, erscheint der Elefant mit seinem außergewöhnlichen Gedächtnis und seiner herausragenden Trägheit in fine als sehr berührendes Tier, als »Moment extremer Fragilisierung, die Kehrseite einer Verhärtung, eine letzte Version der Mobilisierung von Gefühlen, alles in allem: eine radikale Version des Protests. $\|^{38}$ In Dezember finden wir kaum Elefanten, nur ihre Vorfahren, die Mammuts (1.12.1941, 29.12.21.999 v. Chr.): Nicht die, die vor drei Millionen Jahren in Afrika lebten, sondern die Wollhaarmammuts mit ihrem fülligen roten 30 bis 40 Zentimeter langen Fell, die sich erst vor 120.000 Jahren an kalte Regionen anpassten und von den »Lappen« der Altsteinzeit gejagt wurden:

Dr.-Ing. Fred Sauer, ehemals Siemens, für die Versuchsabteilung des Heereswaffenamtes tätig, untersucht die Anatomie von Mammuten. Ließ sich aus den kurzen Rümpfen und gedrungenen Körpern dieser erfahrenen Riesen der Kaltsteppe (die es mit ihren staubigen, immerwährenden, extrem kalten Ostwinden im Jahr 1941 nicht

37 Grégory Cormann/ Jeremy Hamers, "Kluge, Adorno et l'indomptable Leni Peickert«, Cahiers du GRM, 5 | 2014. [https://journals.openedition.org/grm/412].

38 Ebd., "un moment de fragilisation extrême, l'envers d'un durcissement, une version ultime de la mobilisation des sentiments, en somme: une version radicale de la protestation." 
mehr gibt) eine winterfeste Panzerwaffe entwickeln? In den gewaltigen Säulenbeinen, so Fred Sauer, wärmte das sauerstoffhaltige Blut, das aus dem Körper dieser Tiere strömte, das verbrauchte kalte Blut, das zum Körper aufstieg. Das war ein Hinweis auf die Möglichkeit, durch doppelte Kreisläufe in den Motoren (einer zur Erwärmung des Gerätes und einer für den Antrieb) eine Aushilfe gegen die Tücke des russischen Winters zu finden. Das Projekt kommt für die Entscheidung in diesem Jahr zu spät. Der Monat Dezember 1941 war durch Zeitarmut charakterisiert. ${ }^{39}$

Über die bewundernswerte vom Techniker beschriebene Naturmaschinerie und die Ressource, die sie 1941 in den Augen der Armee darstellen, hinaus, beeindrucken diese Exemplare durch ihr Anpassungs- und Überlebenspotenzial in Bedingungen extremer Kälte. Sie verkörpern auch hier eine positive Form des Widerstands gegen die Eiszeit, des Gefühls eingenommen - eine Form des Widerstands, die Kluge durch seine Fiktionen zu reaktivieren sucht. Die unermüdliche Gemeinschaft der Milliarden von Milben ist dessen aktive, weniger spektakuläre, quasi unsichtbare, aber vor Leben und Leistungsfähigkeit wimmelnde Form. Es sind diese Parasiten, die, jenseits jeglicher Rationalität, den Blickwechsel (Wechsel der Blickrichtung) vollziehen, den die Lektüre der Fotos Richters erfordert.

Für die zwei Zeitgenossen des Nationalsozialismus, die Kluge und Richter in ihrer Kindheit waren, ist eines der wesentlichen Anliegen von Dezember auch die Aufarbeitung dieser Geschichte und des auf sie zu werfenden Blicks. Die Aporien des Winters 1941 wiederholen sich auf gewisse Weise wenige Tage vor dem Abtritt Michail Gorbatschows zu Weihnachten 1991. Das Ende der Geschichte vom 2. Dezember: "Da wußten wir: es ist aus«, scheint seit den ersten Dezembertagen die unselige Seite des Monats zu bezeugen. Aber ohne in die Debatte über den Gorbatschewismus einzusteigen, wo sich die Deutungen von Erfolg und Scheitern teilweise überschneiden - das Scheitern des Gorbatchewismus authentifiziert paradoxerweise dessen Erfolg -, gilt es vor allem, die Idee einer verpassten Gelegenheit wahrzunehmen. Davon berichtet auch, auf tragikomische Weise und mit fünfzig Jahren Abstand nach hinten, die Geschichte des 3. Dezember 1931, die ebenfalls von einer wahren Begebenheit inspiriert ist, an der die zwei Künstler indirekt beteiligt sind. Kluge gesteht, dass er diese auf Anfrage von Richter neu schreiben musste:

Kluge: Gerhard Richter kann sehr streng werden, wenn ihm eine Geschichte nicht gefällt.

Wie hört sich ein strenger Gerhard Richter an?

Kluge: Er sagt, das passt nicht, das musst du neu schreiben. Aber nehmen Sie mal die nächste Geschichte: 3. Dezember 1931. Eisregen über Mecklenburg. Eine wahre Geschichte: Hitler in seinem Mercedes wäre auf der Rückfahrt von der Goebbelsschen

39 D, S. 7. 
Hochzeitsgesellschaft beinahe auf einer Eisstraße mit einem Wagen zusammengestoßen und verunglückt. Damals waren Richter und ich schon in den Bäuchchen unserer Mütter. Wir wären also unter Umständen ohne Hitler geboren worden. Da waren 40 Zentimeter zwischen Zusammenprall und Nicht-Zusammenprall.

Schicksal?

Kluge: Nein. Der Teufel hat die Hand dazwischen gehalten. ${ }^{40}$

$\mathrm{Ob}$ man sie isoliert oder in der Konstellation, die sie besonders mit denen des 2. und 8. Dezember bildet, betrachtet, gibt die Geschichte des 3. Dezembers Aufschluss über den modus operandi der zwei Schöpfer. Indem sie Intimes, Zeitgeschehen und Geschichte verbinden, brechen sie »die strikte Chronologie durch ein Netz von Anachronismen, die aus eigenen Montagen oder Konstruktionen von Hypothesen stammen. $\aleph^{41}$ Die Quellen geben für einen Autounfall Hitlers unterschiedliche Daten zwischen März 1930 und Dezember 1931 an. Nichts bestätigt, dass er sich nach der Hochzeit Goebbels ereignet hat, von der man hingegen mit Sicherheit weiß, dass sie am 19. Dezember 1931 stattfand und nicht am 3. Dezember. Dennoch widersprechen weder der Anachronismus noch der Irrealis (»Ich [...] wäre beinahe geboren worden, ohne daß Hitler ein Stück Zukunft hätte.«), noch der anekdotische Ton, der Historie. Kluges Schreiben, das bestrebt ist, die Sättigung oder Derealisation der Geschichte zu vermeiden, indem er die Geschichte auf die Probe der Fiktion stellt, kreuzt das stete Bemühen Richters, die faktuale Materie nicht zu vernachlässigen und sich ihr so nah wie möglich anzunähern, um sich besser von ihr zu distanzieren. Diese Vorgehensweise gleicht jener des amerikanischen Historikers Henry A. Turner (1932-2006), einem Altersgenossen von Richter und Kluge, der 1989 in seinem Werk Geißel des Jahrhunderts. Hitler und seine Hinterlassenschaften eine kontrafaktische Entwicklung Deutschlands imaginiert, die hätte stattfinden können, wenn Hitler 1930 seinem Autounfall erlegen wäre. Der kurze Dialog, möglicherweise zwischen Kluge und Richter, der die Erzählung des 3. Dezember unterbricht, lässt die Frage unbeantwortet, ob es Vorsehung war, dass sich die beiden Autos verfehlten. Mehr als »die Hand des Teufels», von der Kluge im Interview spricht, muss man im 3. Dezember einen sverpassten Unfall sehen, eine Potenzialität, die sich leider in Nichts aufgelöst hat.

Kluge webt in den Stoff seiner Narration unterschiedliche Perspektiven und textuelle oder visuelle Genres ein. Diese wirken als Sinnesreize und auf Sinn hin, aber es gebührt dem Leser, die Leerstellen zwischen ihnen auszufüllen und sie in Verbindung zu bringen (sie zusammenzusetzen, zu montieren, zu kommentie-

40 "Gerhard Richter zeigt den Winter" (Anm. 2).

41 Georges Didi-Huberman, Quand les images prennent position. L'œeil de l'Histoire, 1, Paris, Les Éditions de Minuit, 2009, S. 21: "la stricte chronologie par un réseau d'anachronismes issus de [leurs] propres montages ou constructions d'hypothèses". 
ren), eine Kohärenz zu schaffen, jeder auf seine Art, mit seinen Methoden und seiner Subjektivität oder seinem Eigensinn. Das Mittel dieser operativen Literatur ist eine nichtlineare und elliptische Narration, wobei der Moment an der Kreuzung der Zeiten verweilt, mit der spezifischen Dichte dessen, was zugleich in der Zukunft und im Gewesenen verankert ist. Dies ist auch ein Ziel des kontinuierlichen Stroms der Fragmente von Dezember: die Zeitschichten zusammenzusetzen, um Wirklichkeitskonstellationen zu schaffen.

Wenn uns das Bild der Konstellation zur Beschreibung der Erzählstruktur Kluges noch angemessener scheint als das des Korallenriffs ${ }^{42}$, dann aufgrund seiner doppelten, physischen und abstrakten Bedeutung, und dem, was sie an Erhellung bringt. Sie scheint dem universalistischen Projekt Kluges und seiner Idee eines 'globalen ‘ Autors, der relationes - in beiderlei Sinne, Erzählungen und Verbindungen - herstellt, zu entsprechen: Ihre Bedeutung erhellt sich erst in der Beziehung der Dinge untereinander, in den Erzählungen und dem Bewusstsein darüber. Weist die Konstellation nach dem Muster des Korallenriffs, ein zufälliges Bild auf - die Konstellation kann glücklich oder unglücklich sein -, überlässt sie den Löwenanteil der Vorstellungskraft, gleich den Linien, die diese zwischen den Sternen zieht, um eine sinnvolle Figur entstehen zu lassen. Die Klugesche Erzählung strebt zum »Himmelsäther», zu dieser »Schwingung», die Sein und Nicht-Sein verbindet, von der in "Wiederkehr des Himmelsäthers « ${ }^{43}$, der zehnten der sechzehn Geschichten, die Kluge Anselm Kiefer 2012 widmet, die Rede ist. Einerseits ermöglicht der Klugesche Lakonismus - d.h. die »radikale Auslöschung jeglicher erzwungenen Harmonie oder störenden stilistischen Ablenkung, mit denen die Fabel zeitweise fabuliert ${ }^{44}$ - die Wahrnehmung der Schrillheit der Zeit. Andererseits ist es Kluge wichtig, wie Kiefer das Ohr für die Hintergrundmusik der Welt zu öffnen, für ihre unterirdischen Akkorde, jenseits der oft unharmonischen Klänge einer lauten und geschwätzigen Epoche.

Bei dem Achtzigjährigen wird ein eigentümlicher Appell an den Himmel laut. Den traurigen Jahrestag des Bombenangriffs auf Aleppo im November 2016 auf seine Weise begehend, wiederholt er die epochenüberschreitende Gefühlsgemeinschaft, «wenn Lebensläufe von der Zeitgeschichte zerrissen ${ }^{45}$ werden.

42 Siehe u. a. Wißkirchen, Regen im Korallenriff - Alexander Kluges »Vierzehn Arten, den Regen zu beschreiben« im Kontext, Hamburg 2013.

43 »Sechzehn Geschichten für Anselm Kiefer/Seize histoires pour Anselm Kiefer«, übersetzt von Hilda Inderwildi/ Vincent Pauval, in: Die Ungeborenen, Paris 2012 (ohne Seitenzahlen).

44 Hilda Inderwildi/ Vincent Pauval, "Entre textes et toiles : métamorphoses klugiennes d'un peintre. À propos de >Onze histoires pour Gerhard Richter«, in: Fario, n ${ }^{\circ}$, Paris automne 2012 - hiver 2013, S. 216: "la suppression radicale de toute harmonie forcée ou diversion stylistique gênante dont la fable s'affublerait."

45 "Avant-propos«, in: Kluge, Chronique des sentiments, Livre II »Inquiétance du temps«, Vincent Pauval (Hg.), übersetzt von Anne Gaudu / Kza Han / Herbert Holl / Arthur Lochmann / Vincent Pauval, Paris, P.O.L., 2018. »Werden Lebensläufe von der Zeitgeschichte 
Kraftvoll und originell, mit einem Klarsinn, von dem René Char sagt, dass er die »die sonnennächste Wunde « ${ }^{46}$ sei, behauptet er erneut die Bedeutung der kritischen Poetik, ihrer Fähigkeit, Zusammenhänge herzustellen, »um zu begreifen, wie die Welt es mit uns meint«. Die Erfahrung der Bombenangriffe auf Halberstadt 1945 verschmilzt mit jenen auf Aleppo 2016: Für die, die sie erleiden, bleibt die "Strategie von unten", die Flucht, die Suche nach einem Ausweg dieselbe. Angesichts des Himmels, diesem Ort, wo sich Himmelsäther wie "Strategie von oben", der Bombenregen, entfalten, spricht der Chronist ein Glaubensbekenntnis aus: Er will »himmelschreiender Kritiker sein«. Indem er die vitale Symbolik der Wolfsmilch wiederaufgreift, erscheint der Achtzigjährige als altes Kind mit einzigartigem Schicksal. Von der fabelhaften "Wölfin« (Lupa), sei es die Prostituierte oder das Tier, ernährt, zeigt sich der Bote "von unten « in seiner Verwandtschaft mit den großen Helden der westlichen Zivilisation. Antike Mythologie, christliche Terminologie und politische Utopie (u. a. jene Immendorffs) verklären sich im Schmelztiegel des Lebens:

"Der Himmel hört auf zu malen und wendet sich der Kritik zu“

Ein Bombengeschwader am frühen Morgen am wie immer gefärbten Himmel begründet das Denken neu. Wäre mein Körper aus Stahl und so biegsam wie eine junge Pappel, ich könnte das Bombenfragment, das mich treffen will, abfedern. So kritisiert der SICH VERÄNDERNDE HIMMEL OBEN, den Körper, die Sinne und den Geist und fordert dringlich den Homo Novus, wie er zuletzt 1917 von den Biokosmisten der russischen Revolution ins Auge gefaßt wurde. Wo Brüder seid Ihr jetzt in meiner Not? Es war genug Zeit, mit Euch in Verbindung zu treten, aber ich war beschäftigt. Ich habe die kristallenen Farben des Himmels abzuzählen versucht. Der Himmel in der Frühe und der in der Abenddämmerung ist in unseren Breiten ein begabter Maler. Einige Sekunden vor meinem Ende (und das meiner Lieben) - und wenn der Einschlag den Nachbarn trifft künftig immerfort - will ich himmelschreiender Kritiker sein. Ich sauge an den Zitzen der Wölfin, um dieses Wundermittel in mich hineinzufüllen, falls mir Zeit bleibt. $^{47}$

Bei Kluge und Richter bedeutet die Intention der Verankerung in einem gemeinsamen Sinn und Empfinden, in einem geteilten und gewissermaßen populären Wissen, zunächst ein Mittel, der Gewalt des Stils und den mit der

zerrissen, ist Poetik kein Kitt, kein Klebestoff und keine Nähnadel. Aber um zu begreifen, wie die Welt es mit uns meint, dafür kann die Poetik Zusammenhänge herstellen."

46 René Char, »Feuillets d’Hypnos« (1943-1944), in: Ders. Poésies/Dichtungen. Bd. I, hg. von Jean-Pierre Wilhelm unter Mitarbeit von Christoph Schwerin. Frankfurt, Fischer, 1959, S. 177.

47 Diese Geschichte ist das zweite Beispiel für "Gegen-Erzählungen«, die auf die Bekämpfung von Allmachtsfantasien (die Phantasmen, die eine funktionale globalisierte Welt gebiert) abzielen, im Vorwort des 2. Buches der französischen Ausgabe der Chronique des sentiments (Anm. 45). 
hartnäckigen Suche nach Originalität verbundenen Konkurrenzverhältnissen zu entkommen. Die Ambition, sich in einem Duo zu verbinden, das sich dem Kollektiv nicht verweigert, lässt sich auch als Suche nach einem "gesteigerten" Geist interpretieren, wobei der Künstler nicht nur über den eigenen und sein eigenes Ausdrucksmittel verfügt, sondern auch über jene seiner »Freunde«. Die Frage liegt nahe, ob dieser Geist nicht auch eine Art pragmatische, durch den Mangel an Sinn legitimierte `Trägheit` und/oder Altersangst kompensiert. Bei beiden Künstlern entspricht ein einfacher, aber vor allem freier und paradoxer Ausdruck dem Recht auf Banalität, auf Alltag, das Herbe und Uneinheitliche, sogar auf Inkohärenz - dies alles im Dienst eines an die Empfindung gebundenen Wissens.

Zusätzlich zu den Zweideutigkeiten des Sinns situiert sich der experimentelle und kurios dokumentarische Charakter des Werkes Dezember in diametralem Gegensatz zu gewissen (inter-)diskursiven Formen der Volksliteratur, die sich als Vektor ideologischer Bildung verstehen. Dies gilt auch für den sozialen und politischen Inhalt des Werks: Kein Gutmenschentum, keine Gewissheit, die nicht zunichtegemacht würde durch Humor und das Primat der Intuition, durch Empfindung oder Gefühl. Aus diesem Blickwinkel knüpft Richter über den Kalender, den Dezember komponiert, auch an sein erstes Künstlerbuch von 1966, polke / richter. richter / polke ${ }^{48}$, an, wo die Mischung der Genres und der Künste, die gegen die klassischen Ideale der damals geltenden Reinheit und Klarheit verstößt, Teil einer Volkskultur ist, die sich vom Gedanken der Abschottung losmacht. Das Nachleben der fragmentarischen und intermedialen Form bezeugt, dass das Experimentelle von Gestern zur Norm von Heute werden kann, dass die Kategorie des Populären sich ausweiten muss zu einer beweglichen Palette abwechslungsreicher und widersprüchlicher Suggestionen.

Dezember demonstriert die Relevanz der Betrachtung der Intermedialität aus dem Blickwinkel des Dissens, weniger in seiner prinzipiellen Negativität als in der ihm inhärenten Möglichkeit, jene Alterität konkret zu erproben, von der die Konzeptualisierung ausgehen kann. Die intermediale Praxis verkörpert so die Erfahrung einer gewissen Form des Rückzugs und des Spiels, die kritische Distanz erlaubt und dazu zwingt, den Status des Werks wie den Platz des Lesers und Betrachters neu zu denken. Es handelt sich bei Kluge und Richter um ein konzeptuelles Paradigma, das die beiden Künstler an die Stelle ehemaliger kritischer Reflexe setzen, wobei sie die Kategorie des Hybriden, in dem der Raum sich nicht mehr nur als Grenze gibt, neu gründen.

Toulouse, 30. Juni 2018

48 polke / richter. richter / polke, galerie h, Hannover, 1. bis 26. März 1966, Katalog, Entwürfe, Fotografien, Dokumente, Köln 2014. 